\author{
N. E. Volkova, K. M. Deryabina,
}

L. Ya. Gavrilova, V. A. Cherepanov

Institute of Natural Sciences, Kuybisheva 48 St., Ekaterinburg

Phone: +79045449884;

E-mail: hope3006@yandex.ru

\title{
Physicochemical properties of the complex oxides in Sm-Ba-Me-Cu-O (Me = Fe, Co) systems
}

The present work was focused on the studies of crystal structure, oxygen nonstoichiometry, thermal expansion of $\mathrm{SmBaCO}_{2-x} \mathrm{Cu}_{x} \mathrm{O}_{6-\delta}$ and $\mathrm{SmBaFe}_{2-x} \mathrm{Cu}_{x} \mathrm{O}_{6-\delta}$. Values of the oxygen content in complex oxides $\mathrm{SmBaCO}_{2-x} \mathrm{Cu}_{x} \mathrm{O}_{6-\delta}$ were determined over a wide temperature range in air using high temperature thermogravimetry and iodometric titration. Also, chemical stability with respect to $\mathrm{Ce}_{0.8} \mathrm{Sm}_{0.2} \mathrm{O}_{1.9}$ and $\mathrm{Zr}_{0.85} \mathrm{Y}_{0.15} \mathrm{O}_{1.93}$ two common solid electrolyte materials used for SOFCs was evaluated.

\footnotetext{
* The work was supported under financial support from RFBR № 13-03-00958 and Ministry of Science and Education of Russian Federation within the limits of the Federal target program "Scientific and scienceeducational cadres of innovative Russia for 2009-2013"
}

(C) Volkova N. E., Deryabina K. M, Gavrilova L. Ya., Cherepanov V. A., 2015

\section{Introduction}

Layered perovskites compounds $\mathrm{AA}^{\prime} \mathrm{B}_{2} \mathrm{O}_{6-\delta}$ wherein $\mathrm{A}$ is a lanthanide partially substituted by alkaline earth metal $\mathrm{A}^{\prime}$, and $\mathrm{B}$ is $3 \mathrm{~d}$-atoms of metal ( $\mathrm{Ti}, \mathrm{Cr}$, $\mathrm{Mn}, \mathrm{Fe}, \mathrm{Co}, \mathrm{Ni}, \mathrm{Cu}$ ), are currently under scrutiny due to the successful combination of their physico-chemical properties ${ }^{1,3}$.

Physico-chemical properties of the oxides formed in systems $\mathrm{LnBaMe}_{2-x} \mathrm{M}_{x} \mathrm{O}_{6-\delta}$, are directly dependent on their crystal structure, the formation that can be sig- nificantly affected by an oxygen content. In this regard, information on the method of production, physico-chemical properties and stability of oxides $\mathrm{AA}^{\prime} \mathrm{B}_{2} \mathrm{O}_{5+\delta}$ under variation of the chemical composition and external thermodynamic conditions today is up-to-date. Therefore, the aim of this paper is to receive, and to analyze the crystallical structure and physico-chemical properties of complex oxide phases forming in the systems $\mathrm{SmBaMe}_{2-x} \mathrm{Cu}_{x} \mathrm{O}_{6-\delta}$ $(\mathrm{Me}=\mathrm{Fe}, \mathrm{Co})$.

\section{The experimental part}

Synthesis of the samples for the study was carried out on glycerol-nitrate technology. To prepare the samples samarium oxide $\mathrm{Sm}_{2} \mathrm{O}_{3}$ and barium carbonate

$\mathrm{BaCO}_{3}$ were used as starting components; they were previously calcined to remove adsorbed moisture and gases, metallic cobalt, and iron oxalate $\mathrm{FeC}_{2} \mathrm{O}_{4} \times 2 \mathrm{H}_{2} \mathrm{O}$, 
as well as nitric acid $\mathrm{HNO}_{3}$ (qualification analytically pure), and glycerol (analytical grade qualification). Cobalt metal was prepared by reduction of corresponding oxides at $500-600^{\circ} \mathrm{C}$ in a stream of hydrogen. Thermogravimetric studies were carried out on the thermobalance STA 409 PC by Netzsch Gmbh., allowing to fixate changes in mass of the sample in dependence of the partial pressure of oxygen and temperature.

Measurements were carried out in static and dynamic modes. In the static mode, the sample (1-2 g), previously weighed in a platinum crucible, was heated to the temperature of the beginning of the measurements, it was held at this temperature for 10 hours to establish equilibrium between the solid and gas phases, and then the temperature was raised again and the constant of the weight of the sample was expected. Investigated temperature range of $300-1100{ }^{\circ} \mathrm{C}$ was held in the heating

\section{Results and Discussion}

Complex oxides $\mathrm{SmBaCo} \mathrm{SuxO}_{6-x}$ :

On glycerol-nitrate technology hard solutions were synthesized compositions $\mathrm{SmBaCo}_{2-x} \mathrm{SuxO}_{6-\delta}$ where $0 \leq x \leq 2$. XRD revealed that single-phase complex oxides $\mathrm{SmBaCo}_{2-x} \mathrm{Su}_{x} \mathrm{O}_{6-\delta}$ form in the composition range $0 \leq h \leq 1.2$.

With minimal substitution of cobalt with copper $(x=0.1)$ a composite oxide is formed, which X-ray is satisfactorily described within the orthorhombic cell (pr. G. Pmmm).

Radiographs of the samples with a high content of copper $(0.2 \leq h \leq 1.2)$ have been indexed within the tetragonal unit cell of the space group P4/mmm. In fig. 1 as an example of a complex oxide radiograph $\mathrm{SmBaCo}_{1.4} \mathrm{Su}_{0.6} \mathrm{O}_{5+\delta}$ is represented. and cooling mode to $100{ }^{\circ} \mathrm{C}$. In the dynamic mode the change in weight was continuously recorded during heating and cooling with a speed of $2{ }^{\circ} \mathrm{C}$ per min from room temperature to $1100{ }^{\circ} \mathrm{C}$. The absolute value of oxygen deficiency was determined by direct reduction samples in hydrogen and iodometric titration methods. For this, the samples were slowly cooled to room temperature. Measurement of the linear coefficient of thermal expansion (LKTR) of ceramic materials is necessary to determine the possibility of obtaining of lasting contact in high-temperature electrochemical devices (such components are electrolytes, electrodes, electrical circuits, sealants, etc.).

Measurements of relative expansion of ceramic bars with increasing temperature were performed on dilatometer DIL402 $\mathrm{C}$ by Netzsch Gmbh in the temperature range $25-1100{ }^{\circ} \mathrm{C}$ with the heating and cooling rate of $5^{\circ}$ per minute.

For all single-phase oxides unit cell parameters of the atomic coordinates were calculated. When the concentration of copper ions is increasing, an increase in parameters and volume of the unit cells of complex oxides $\mathrm{SmBaCo}_{2-x} \mathrm{Cu}_{x} \mathrm{O}_{6-\delta}$ is observed. Such dependence can be explained in terms of size effects. Substitution of cobalt ions $\left(r_{\mathrm{C}^{3+}} / r_{\mathrm{C}^{4+}}=0.75 /\right.$ $0.67 \AA$, cn 6) with large size copper ions $\left(r_{\mathrm{Cu}^{2+}} / r_{\mathrm{Cu}^{3+}}=0.87 / 0.68 \AA\right.$, cn 6$)[4]$ leads to a gradual increase in the bond lengths in the B-O, and as a consequence, increase in the size of the cell unit.

The phase composition of the samples $\mathrm{SmBaCo}_{2-x} \mathrm{Cu}_{x} \mathrm{O}_{6-\delta}$, outside the homogeneity region is presented in Table 1. 


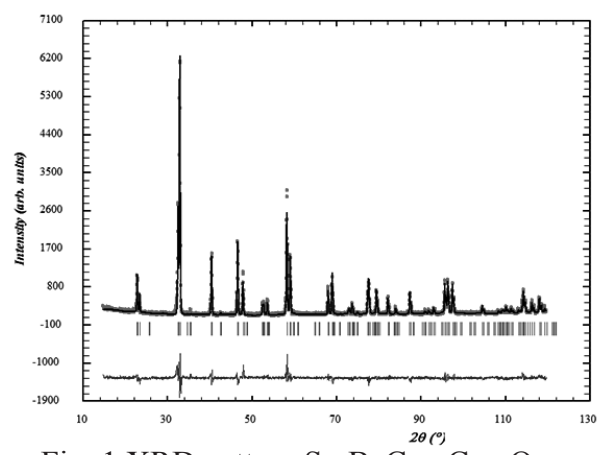

Fig. 1 XRD pattern $\mathrm{SmBaCo}_{1.4} \mathrm{Cu}_{0.6} \mathrm{O}_{6-\delta}$, treated by the method of Rietveld. Points are experimental data; solid top shedding is theoretical spectrum; solid bottom line is the difference between the experimental data and the theoretical curve.

Table 1

\begin{tabular}{c|c}
$\begin{array}{c}\text { Nominal } \\
\text { composition of the } \\
\text { samples }\end{array}$ & $\begin{array}{c}\text { Phase composition } \\
\text { of the samples }\end{array}$ \\
\cline { 1 - 1 } $\mathrm{SmBaCo}_{0.7} \mathrm{Cu}_{1.3} \mathrm{O}_{6-\delta}$ & $\mathrm{SmBaCo}_{0.8} \mathrm{Cu}_{1.2} \mathrm{O}_{6-\delta}$ \\
\cline { 1 - 2 } $\mathrm{SmBaCo}_{0.6} \mathrm{Cu}_{1.4} \mathrm{O}_{6-\delta}$ & $\mathrm{Sm}_{3} \mathrm{Ba}_{3}(\mathrm{Cu}, \mathrm{Co})_{6} \mathrm{O}_{14-2 \delta}$ \\
\hline $\mathrm{SmBaCo}_{0.4} \mathrm{Cu}_{1.6} \mathrm{O}_{6-\delta}$ & $\mathrm{Sm}_{3} \mathrm{Ba}_{3}(\mathrm{Cu}, \mathrm{Co})_{6} \mathrm{O}_{14-2 \delta}$ \\
\hline $\mathrm{SmBaCo}_{0.2} \mathrm{Cu}_{1.8} \mathrm{O}_{6-\delta}$ & $\mathrm{Sm}_{3} \mathrm{Ba}_{3} \mathrm{Cu}_{6} \mathrm{O}_{14-2 \delta}$
\end{tabular}

The compounds of nominal composition $\mathrm{Sm}_{3} \mathrm{Ba}_{3}(\mathrm{Cu}, \mathrm{Co})_{6} \mathrm{O}_{14-2 \delta}$ are solid solutions $\mathrm{SmBa}_{2-x} \mathrm{Sm}_{x}(\mathrm{Cu}, \mathrm{Co})_{3} \mathrm{O}_{7-\delta}{ }^{4-6}$. The crystal structure of these compounds has been described in terms of the tetragonal unitcellwith tripleoption with $\left(a_{p} \times a_{p} \times 3 a_{p}\right)$ space group $P 4 / \mathrm{mmm}$.

Figure 2 shows the temperature dependence of the oxygen content of complex oxides $\mathrm{SmBaCo}_{2-x} \mathrm{Cu}_{x} \mathrm{O}_{6-\delta}$.

With the introduction of copper in cobalt sublattice a decrease in the value of the oxygen content is observed. This is due to the fact that the copper becomes totally or partially the electron acceptor $\left(\mathrm{Cu}_{\mathrm{Co}}^{\prime}\right)\left(\mathrm{EO}_{\mathrm{Cu}}=1.75\right)$ and contributes to the oxygen vacancy $\left(V_{O}^{*}\right)$ and/or electron holes.

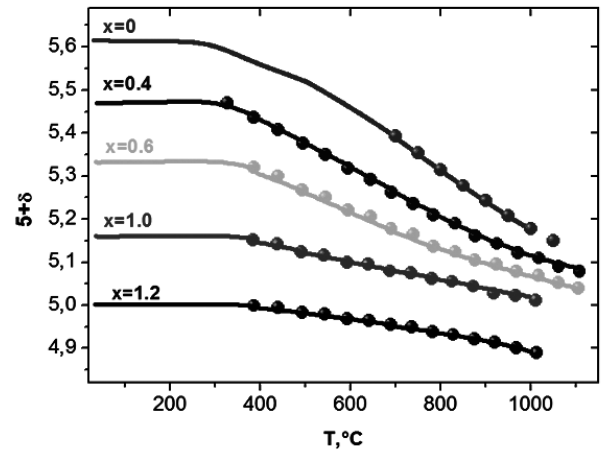

Fig. 2. The dependence of the oxygen content of the temperature for $\mathrm{SmBaCo}_{2-x} \mathrm{CuxO}_{6-\delta}$

Measuring of the relative increase in the size of the samples $\mathrm{SmBaCo}_{2-x} \mathrm{Cu}_{x} \mathrm{O}_{6-\delta}$ $(x=0 ; 0.4 ; 0.6)$ was performed in air in the temperature range of $25-1100{ }^{\circ} \mathrm{C}$ at a rate of heating and cooling $2^{\circ}$ per minute.

For this, the powders of the oxides were compressed under a pressure of 60 to $80 \mathrm{bar}$ in the form of bars of size $2 \times 4 \times 20 \mathrm{~mm}$ via a hydraulic press. The resulting bars were sintered in air at $1050-1200^{\circ} \mathrm{C}$ for 14 hours and then slowly cooled to room temperature at a rate $100^{\circ}$ per hour.

The density of the sintered layered perovskites of samarium-barium $\mathrm{SmBa}-$ $\mathrm{Co}_{2-x} \mathrm{Cu}_{x} \mathrm{O}_{6-\delta}$ is at least $90 \%$ of the calculated from the X-ray data.

Monotonic character of dilatometric dependencies for complex oxides Sm$\mathrm{BaCo}_{2-x} \mathrm{Cu}_{x} \mathrm{O}_{6-\delta}$ indicates the absence of phase transitions. It has been established that the value of the CTE decreases with increasing concentration of copper in $\mathrm{SmBaCo}_{2-} x \mathrm{Cu}_{x} \mathrm{O}_{6-\delta}$.

Chemical compatibility of complex oxides $\mathrm{SmBaCo}_{2-x} \mathrm{Su}_{x} \mathrm{O}_{6-\delta}(x=0$; $0.2 ; 0.4 ; 1.0)$ with respect to the material of the electrolyte $\mathrm{Ce}_{0.8} \mathrm{Sm}_{0.2} \mathrm{O}_{2-\delta}$ and $\mathrm{Zr}_{0.85} \mathrm{Y}_{0.15} \mathrm{O}_{2-\delta}$ were studied by contact annealing at $900^{\circ} \mathrm{C}, 1000^{\circ} \mathrm{C}, 1050^{\circ} \mathrm{C}$, and $1100^{\circ} \mathrm{C}$ in air. 
$\mathrm{XRD}$ revealed that complex oxides $\mathrm{SmBaCo}_{2-x} \mathrm{Su}_{x} \mathrm{O}_{5+\delta} \mathrm{cx}=0-0.4$ do not react with a stabilized ceria oxide. And by annealing of the sample $\mathrm{SmBaCoSuO}{ }_{6-\delta}$ with $\mathrm{Ce}_{0.8} \mathrm{Sm}_{0.2} \mathrm{O}_{2-\delta}$ at a temperature of $1000{ }^{\circ} \mathrm{C}$, the diffraction patterns of the annealed mixtures present reflexes related to the cobaltites and cuprates samarium and barium.

The electrolyte based on zirconia at a temperature of $900{ }^{\circ} \mathrm{C}$ is reacted with all samples of $\mathrm{SmBaCoSuO}_{5+\delta}$. As the main impurity phase in the diffraction patterns of the annealed mixtures present reflexes related to $\mathrm{BaZrO}_{3-\delta}$.

Complex oxides $\mathrm{SmBaFe} \mathrm{Su}_{2-x} \mathrm{O}_{6-\delta}$ :

Solid solutions $\mathrm{SmBaFe}_{2-x} \mathrm{Su}_{x} \mathrm{O}_{6-\delta}$, where $0.5 \leq x \leq 1.5$ with 0.1 step were synthesized by the glycerol-nitrate technol- ogy. According to X-ray data, solid solutions $\mathrm{SmBaFe}_{2-x} \mathrm{Su}_{x} \mathrm{O}_{6-\delta}$ with $0.7 \leq x \leq 1.3$ are single-phase. Radiographs of oxides $\mathrm{SmBaFe}_{2-x} \mathrm{Su}_{x} \mathrm{O}_{6-\delta}$ are satisfactorily described in terms of the tetragonal unit cell of the space group $P 4 / \mathrm{mmm}$. Example radiographs of solid solution $\mathrm{SmBaFe}_{1.2} \mathrm{Su}_{0.8} \mathrm{O}_{6-\delta}$, treated by the method of full-profile Rietveld analysis are shown in Fig. 3.

For all single-phase oxides there were calculated unit cell parameters of the atomic coordinates. It was found that an increase in the concentration of copper in $\mathrm{SmBaFe}_{2-x} \mathrm{Su}_{x} \mathrm{O}_{6-\delta}$ leads to a gradual increase in the parameters and the unit cell volume, which can be explained in terms of the size factor.

values of oxygen content in the complex oxides formed in systems $\mathrm{Sm}-\mathrm{Ba}-\mathrm{Co}-\mathrm{Cu}-\mathrm{O}$ in a wide temperature range in air. It was found that the introduction of copper reduces the oxygen content in $\mathrm{SmBaCo}_{2-x} \mathrm{Me}_{x} \mathrm{O}_{6-\delta}$;

4. Coefficients of thermal expansion (CTE) of solid solutions $\mathrm{SmBaCo}_{2-}$ ${ }_{x} \mathrm{Cu}_{x} \mathrm{O}_{6-\delta}(x=0 ; 0.4 ; 0.6)$ are calculated. It

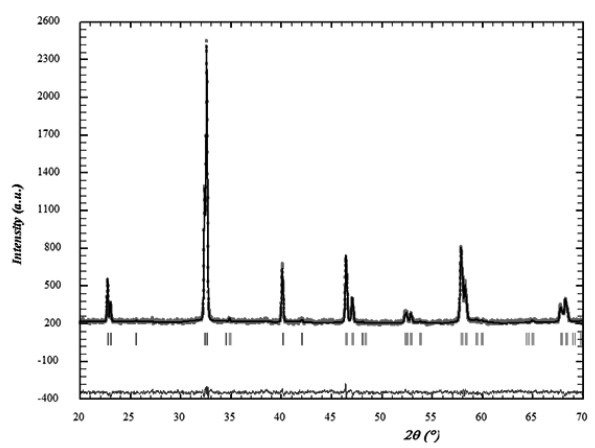

Fig. $3 \mathrm{X}$-ray data for $\mathrm{SmBaFe}_{1.2} \mathrm{Su}_{0.8} \mathrm{O}_{6-\delta}$, treated by the method of Rietveld 
is shown that the magnitude of the CTE decreases with increasing concentration of copper in the samples;

5. The chemical compatibility of complex oxides with the material of the solid electrolyte $\left(\mathrm{Ce}_{0.8} \mathrm{Sm}_{0.2} \mathrm{O}_{2-\delta}\right.$ and $\left.\mathrm{Zr}_{0.85} \mathrm{Y}_{0.15} \mathrm{O}_{2-\delta}\right)$ is researsched at $900 \leq \mathrm{T}$, ${ }^{\circ} \mathrm{C} \leq 1100$ and $\mathrm{Po}_{2}=0.21 \mathrm{~atm}$. It has been shown that solid solutions $\mathrm{SmBaCo}_{2}$ ${ }_{x} \mathrm{Me}_{x} \mathrm{O}_{6-\delta}$ interact with stabilized zirconia at a temperature of $900{ }^{\circ} \mathrm{C}$ and do not interact with the stabilized cerium oxide to a temperature not higher than $900-1100^{\circ} \mathrm{C}$ depending on the concentration of copper ions.

1. Maignan A., Martin C., Pelloquin D., Nguyen N., Raveau B. Structural and Magnetic Studies of Ordered Oxygen - Deficient Perovskites LnBaCo2O5, Closely Related to the "112" Structure. Journal of Solid State Chemistry. 1999;142:247-260. DOI: 10.1006/jssc. 1998.7934.

2. Anderson P., Kirk C., Knudsen J., Reaney I., West A. Structural characterization of ReBaCo2O6- $\delta$ phases (RE $=\mathrm{Pr}, \mathrm{Nd}, \mathrm{Sm}, \mathrm{Eu}, \mathrm{Gd}, \mathrm{Tb}, \mathrm{Dy}, \mathrm{Ho})$. Journal of Solid State Sciences 2005;7:1149-1156. DOI: 10.1016/j.solidstatesciences.2005.03.004.

3. Zhang K., Ge L., Shao Z., Lio S. Synthesis, characterization and evaluation of cation-ordered $\mathrm{LnBaCo} 2 \mathrm{O} 5+\delta$ as materials of oxygen permiation membranes and cathodes of SOFCs. Acta Materialia. 2008;56(17):4876-4889. DOI: 10.1016/j. actamat.2008.06.004.

4. Shannon R. D. Revised effective ionic radii and systematic studies of interatomic distances in halides and chalcogenides. Acta Cryst. 1976;32A:751-767.

5. Kin N., Shivashankar S., Umarji A., Yelon W., Malik S. Effect of synthesis route on the structure of $\mathrm{Nd} 3-\mathrm{xBa} 3+\mathrm{xCu} 6 \mathrm{O} 14-2 \delta(\mathrm{x}=0,1)$ system: a neutron diffraction study. Solid State Comm. 2002;122(1-2):99-104. DOI: 10.1016/S0038-1098(01)00521-X.

6. Wende C., Schupp B., Krabbes C. Phase equilibria and primary crystallisation field for $\mathrm{Sm}_{1+y} \mathrm{Ba}_{2-y} \mathrm{Cu}_{3} \mathrm{O}_{7}$ at various $\mathrm{p}(\mathrm{O} 2)$. J.Alloys and Comp. 2004;381(1-2):320-326. DOI: 10.1016/j.jallcom.2004.04.093. 
Н. Е. Волкова, К. М. Дерябина, Л. Я. Гаврилова, В. А. Черепанов Институт естественных наук, Екатеринбург, ул. Куйбышева, 48, тел.: +7 (904) 544-98-84 E-mail: hope3006@yandex.ru

\section{Физико-химические свойства сложных оксидов в системах $\mathrm{Sm}-\mathrm{Ba}-\mathrm{Me}-\mathrm{Cu}-0$ $(\mathrm{Me}=\mathrm{Fe}, \mathrm{Co})^{*}$}

Определены области гомогенности твердых растворов $\mathrm{SmBaFe} \mathrm{Cu}_{2-x} \mathrm{O}_{6-\delta}$ $(0,7 \leq x \leq 1,3)$ и $\mathrm{SmBaCo}_{2-x} \mathrm{Cu}_{x} \mathrm{O}_{6-\delta}(0 \leq x \leq 1,2)$ на воздухе и изучена их кристаллическая структура. Построены зависимости параметров элементарных ячеек от состава и уточнены координаты позиций атомов. Методами высокотемпературной термогравиметрии и йодометрического титрования определены значения содержания кислорода в сложных оксидах $\mathrm{SmBaCo}_{2-x} \mathrm{Cu}_{x} \mathrm{O}_{6-\delta}$ в широком интервале температур на воздухе. Рассчитаны коэффициенты термического расширения (KTP) твердых растворов $\mathrm{SmBaCO}_{2-x} \mathrm{Cu}_{x} \mathrm{O}_{6-\delta}(x=$ $0 ; 0,4 ; 0,6)$ при $\mathrm{PO}_{2}=0,21$ атм. Исследована химическая совместимость оксидов $\mathrm{SmBaCo}_{2-x} \mathrm{Me}_{x} \mathrm{O}_{5+\delta}(\mathrm{Me}=\mathrm{Fe}, \mathrm{Ni}, \mathrm{Cu})$ с материалом твердого электролита $\left(\mathrm{Ce}_{0.8} \mathrm{Sm}_{0.2} \mathrm{O}_{2-\delta}\right.$ и Zr $\left.{ }_{0.85} \mathrm{Y}_{0.15} \mathrm{O}_{2-\delta}\right)$ при $900 \leq \mathrm{T}$, ${ }^{\circ} \mathrm{C} \leq 1100$.

* Работа выполнена при финансовой поддержке гранта РФФИ (проект № 13-03-00958) и Министерства образования и науки РФ в рамках федеральной целевой программы «Научные и научно-педагогические кадры инновационной России» на 2009-2013 годы.

(C) Волкова Н. Е., Дерябина К. М., Гаврилова Л. Я., Черепанов В. А., 2015

\section{Введение}

Слоистые перовскитоподобные соединения $\mathrm{AA}^{\prime} \mathrm{B}_{2} \mathrm{O}_{6-\delta}$, где $\mathrm{A}-$ лантаноид, частично замещенный на щелочноземельный металл $\mathrm{A}^{\prime}$, a $\mathrm{B}$ атомы 3d-металла ( $\mathrm{Ti}, \mathrm{Cr}, \mathrm{Mn}, \mathrm{Fe}, \mathrm{Co}$, $\mathrm{Ni}, \mathrm{Cu})$, в настоящее время являются объектом пристального внимания благодаря удачному сочетанию их физикохимических свойств [1-3].

Физико-химические свойства оксидов, образующихся в системах $\mathrm{LnBaMe}_{2-x} \mathrm{M}_{x} \mathrm{O}_{6-\delta}$, непосредственно за- висят от их кристаллической структуры, на формирование которой существенное влияние оказывает содержание кислорода. В связи с этим информация о методах получения, физико-химических свойствах и стабильности оксидов $\mathrm{AA}^{\prime} \mathrm{B}_{2} \mathrm{O}_{5+\delta}$ при варьировании химического состава и внешних термодинамических условий на сегодняшний день является актуальной.

Поэтому целью настоящей работы явилось получение, исследование 
кристаллической структуры и физико-химических свойств сложнооксид- ных фаз, образующихся в системах $\mathrm{SmBaMe}_{2-x} \mathrm{Cu}_{x} \mathrm{O}_{6-\delta}(\mathrm{Me}=\mathrm{Fe}, \mathrm{Co})$.

\section{Экспериментальная часть}

Синтез образцов для исследования осуществляли по глицерин-нитратной технологии. Для приготовления образцов в качестве исходных компонентов использовали оксид самария $\mathrm{Sm}_{2} \mathrm{O}_{3}$ и карбонат бария $\mathrm{BaCO}_{3}$, предварительно прокаленные для удаления адсорбированной влаги и газов, металлический кобальт и оксалат железа $\mathrm{FeC}_{2} \mathrm{O}_{4} \times 2 \mathrm{H}_{2} \mathrm{O}$, а также азотную кислоту $\mathrm{HNO}_{3}$ (квалификация ч.д.а.) и глицерин (квалификация ч.д.а.). Металлический кобальт получали восстановлением из соответствующего оксидов при 500-600 들 токе водорода.

Термогравиметрические исследованияпроводилинатермовесах STA409PC фирмы Netzsch Gmbh., позволяющих фиксировать изменения массы образца в зависимости от парциального давления кислорода и температуры.

Измерения проводили в статическом и динамическом режимах. В статическом режиме образец (навеска 1-2 г), предварительно взвешенный в платиновом тигле, нагревали до температуры начала измерений, выдерживали в течение 10 ч до установления равновесия между твердой и газовой фазами, затем повышали температуру и снова дожидались постоянства массы образца. Исследуемый интервал температур 300-1100 ${ }^{\circ} \mathrm{C}$ на воздухе проходили в режиме нагревания и охлаждения через $100^{\circ}$. В динамическом режиме непрерывно фиксировали изменение массы образца при нагревании и охлаждении со скоростью 2 \%мин от комнатной температуры до $1100{ }^{\circ} \mathrm{C}$ на воздухе.

Абсолютное значение кислородного дефицита определяли методами прямого восстановления образцов в токе водорода и йодометрического титрования. Для этого образцы были медленно охлаждены до комнатной температуры.

Измерение линейного коэффициента термического расширения (ЛКТР) керамических материалов необходимо для определения возможности получения прочного контакта составляющих высокотемпературного электрохимического устройства (таковыми составляющими являются электролиты, электроды, токопроходы, герметики и др.).

Измерения относительного расширения керамических брусков с увеличением температуры проводили на дилатометре DIL402 C фирмы Netzsch Gmbh на воздухе в температурном интервале $25-1100{ }^{\circ} \mathrm{C}$ со скоростью нагрева и охлаждения 5 \%мин.

\section{Результаты и обсуждение}

Сложные оксиды $\mathrm{SmBaCo}{ }_{2-x} \mathrm{Cu}_{x} \mathrm{O}_{6-\delta}$

По глицерин-нитратной технологии, были синтезированы твердые растворы составов $\mathrm{SmBaCo}_{2-x} \mathrm{Cu}_{x} \mathrm{O}_{6-\delta}$, где $0 \leq x \leq 2$.
По данным РФА установлено, что однофазные сложные оксиды $\mathrm{SmBaCo}_{2-x} \mathrm{Cu}_{x} \mathrm{O}_{6-\delta}$ образуются в интервале составов $0 \leq x \leq 1,2$. 
При минимальном замещении кобальта медью $(x=0,1)$ образуется сложный оксид, рентгенограмма которого удовлетворительно описывается в рамках орторомбической ячейки (пр. гр. Ртmm).

Рентгенограммы образцов с большим содержанием меди $(0,2 \leq x \leq$ $1,2)$ были проиндексированы в рамках тетрагональной ячейки пространственной группы $P 4 / m m m$. На рис. 1 в качестве примера представлена рентгенограмма сложного оксида $\mathrm{SmBaCo}_{1,4} \mathrm{Cu}_{0,6} \mathrm{O}_{5+\delta}$. Для всех однофазных оксидов были рассчитаны параметры элементарной ячейки и координаты атомов. При увеличении концентрации ионов меди наблюдается увеличение параметров и объема элементарных ячеек сложных оксидов $\mathrm{SmBaCo}_{2-x} \mathrm{Cu}_{x} \mathrm{O}_{6-\delta}$. Такие зависимости можно объяснить с точки зрения размерных эффектов. Замещение ионов кобальта $\left(r_{\mathrm{Co}^{3+}} / r_{\mathrm{Co}^{4+}}=0,75 / 0,67 \AA\right.$, к.ч. 6) большими по размеру ионами меди $\left(r_{\mathrm{Cu}^{2+}} / r_{\mathrm{Cu}^{3+}}=0,87 / 0,68 \AA\right.$, к.ч. 6)

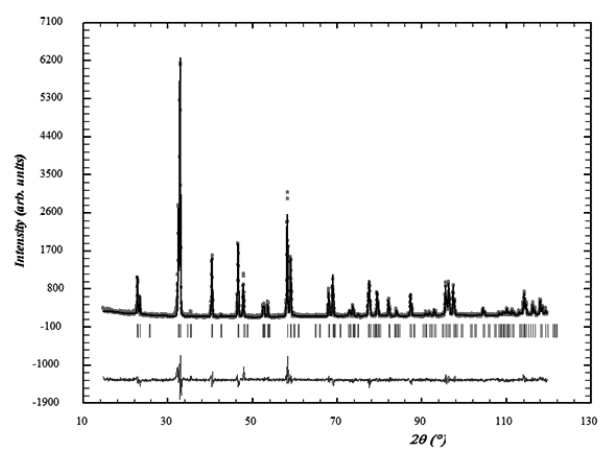

Рис. 1. Рентгенограмма $\mathrm{SmBaCo}_{1.4} \mathrm{Cu}_{0.6} \mathrm{O}_{6-\delta}$, обработанная по методу Ритвелда.

Точки - данные эксперимента; сплошная верхняя линяя - теоретический спектр; сплошная нижняя линия - разница между экспериментальными данными и теоретической кривой
[4] приводит к постепенному увеличению длины связи В-О и, как следствие, увеличению размеров элементарной ячейки.

Фазовый состав образцов $\mathrm{SmBaCo}_{2-x} \mathrm{Cu}_{x} \mathrm{O}_{6-\delta}$, находящихся за пределами области гомогенности, представлен в таблице 1.

Таблица 1

Фазовый состав образцов

\begin{tabular}{c|c}
$\begin{array}{c}\text { Номинальный } \\
\text { состав образцов }\end{array}$ & $\begin{array}{c}\text { Фазовый состав } \\
\text { образцов }\end{array}$ \\
\hline $\mathrm{SmBaCo}_{0,7} \mathrm{Cu}_{1,3} \mathrm{O}_{6-\delta}$ & $\mathrm{SmBaCo}_{0,8} \mathrm{Cu}_{1,2} \mathrm{O}_{6-\delta}$ \\
$\mathrm{SmBaCo}_{0,6} \mathrm{Cu}_{1,4} \mathrm{O}_{6-\delta}$ & $\mathrm{Sm}_{3} \mathrm{Ba}_{3}(\mathrm{Cu}, \mathrm{Co})_{6} \mathrm{O}_{14-2 \delta}$ \\
\hline $\mathrm{SmBaCo}_{0,4} \mathrm{Cu}_{1,6} \mathrm{O}_{6-\delta}$ & $\mathrm{Sm}_{3} \mathrm{Ba}_{3}(\mathrm{Cu}, \mathrm{Co})_{6} \mathrm{O}_{14-2 \delta}$ \\
\hline $\mathrm{SmBaCo}_{0,2} \mathrm{Cu}_{1,8} \mathrm{O}_{6-\delta}$ & $\mathrm{Sm}_{3} \mathrm{Ba}_{3} \mathrm{Cu}_{6} \mathrm{O}_{14-2 \delta}$ \\
\hline $\mathrm{SmBaCu}_{2} \mathrm{O}_{6-\delta}$ & сол- \\
Соединения
\end{tabular}
става $\mathrm{Sm}_{3} \mathrm{Ba}_{3}(\mathrm{Cu}, \mathrm{Co})_{6} \mathrm{O}_{14-2 \delta}$ являются твердыми растворами типа $\mathrm{SmBa}_{2-x} \mathrm{Sm}_{x}(\mathrm{Cu}, \mathrm{Co}) 3 \mathrm{O}_{7-\delta}$ [4-6]. Кристаллическая структура данных соединений была описана в рамках тетрагональной ячейки с утроенным параметром с $\left(a_{p} \times a_{p} \times 3 a_{p}\right)$ пространственной группы $P 4 / \mathrm{mmm}$.

На рис. 2 представлены температурные зависимости содержания кислорода для сложных оксидов $\mathrm{SmBaCo}_{2-x} \mathrm{Cu}_{x} \mathrm{O}_{6-\delta}$.

При внедрении меди в подрешетку кобальта наблюдается понижение величины содержания кислорода. Это связано с тем, что медь становится полностью или частично акцептором электронов $\left(\mathrm{Cu}_{\mathrm{Co}}^{\prime}\right)(Э \mathrm{OCu}=1,75)$ и способствует возникновению кислородных вакансий $\left(V_{O}^{*}\right)$ и/или электронных дырок.

Измерения относительногоувеличения размера образцов $\mathrm{SmBaCo}_{2-x} \mathrm{Cu}_{x} \mathrm{O}_{6-\delta}$ $(x=0 ; 0,4 ; 0,6)$ проводили на воздухе в температурном интервале $25-1100{ }^{\circ} \mathrm{C}$ 


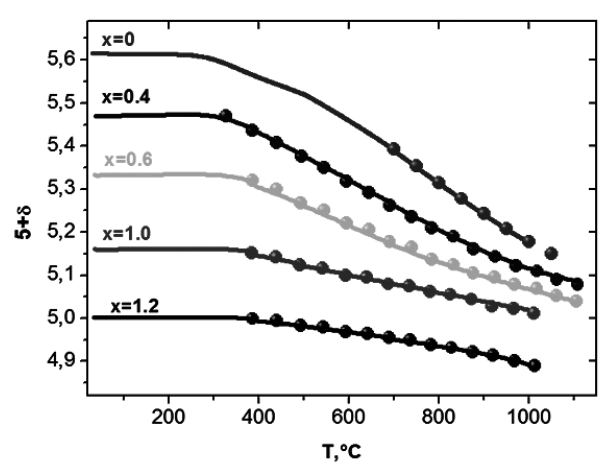

Рис. 2. Зависимости изменения содержания кислорода от температуры на воздухе для $\mathrm{SmBaCo}_{2-x} \mathrm{Cu}_{x} \mathrm{O}_{6-\delta}$

со скоростью нагрева и охлаждения $2 \%$ мин.

Для этого порошки исследуемых оксидов прессовали под давлением 60-80 бар в форме брусков размером $2 \times 4 \times 20$ мм с помощью гидравлического пресса. Полученные бруски спекали на воздухе при $1050-1200{ }^{\circ} \mathrm{C}$ в течение 14 ч и затем медленно охлаждали до комнатной температуры со скоростью $100 \%$ час.

Плотность спеченных слоистых перовскитов самария-бария $\mathrm{SmBaCo}_{2-x} \mathrm{Cu}_{x} \mathrm{O}_{6-\delta}$ составляет не менее $90 \%$ от рассчитанной из рентгенографических данных.

Монотонный характер дилатометрических зависимостей для сложных оксидов $\mathrm{SmBaCo}_{2-x} \mathrm{Cu}_{x} \mathrm{O}_{6-\delta}$ свидетельствует об отсутствии фазовых переходов. Установлено, что величина КТР уменьшается при увеличении концентрации меди в $\mathrm{SmBaCo}_{2-x} \mathrm{CuxO}_{6-\delta}$.

Химическая совместимость сложных оксидов $\mathrm{SmBaCo}_{2-x} \mathrm{Cu}_{x} \mathrm{O}_{6-\delta}(x=0$; $0,2 ; 0,4 ; 1,0)$ по отношению к материалу электролита $\mathrm{Ce}_{0,8} \mathrm{Sm}_{0,2} \mathrm{O}_{2-\delta}$ и $\mathrm{Zr}_{0,85} \mathrm{Y}_{0,15} \mathrm{O}_{2-\delta}$ изучена методом контактных отжигов при температурах $900{ }^{\circ} \mathrm{C}, 1000{ }^{\circ} \mathrm{C}$, $1050{ }^{\circ} \mathrm{C}, 1100{ }^{\circ} \mathrm{C}$ на воздухе.
По данным РФА установлено, что сложные оксиды $\mathrm{SmBaCo}_{2-x} \mathrm{Cu}_{x} \mathrm{O}_{5+\delta} \mathrm{cx}=$ $=0-0,4$ со стабилизированным оксидом церия не реагируют. А при отжиге образца $\mathrm{SmBaCoCuO}_{6-\delta}$ c $\mathrm{Ce}_{0,8} \mathrm{Sm}_{0,2} \mathrm{O}_{2-\delta}$ уже при температуре $1000{ }^{\circ} \mathrm{C}$ на дифрактограммах отожженных смесей присутствовали рефлексы, относящиеся к кобальтитам и купратам самария и бария.

Электролит на основе диоксида циркония уже при температуре $900{ }^{\circ} \mathrm{C}$ взаимодействует со всеми образцами $\mathrm{SmBaCoCuO}_{5+\delta}$. В качестве основной примесной фазы на дифрактограммах отожженных смесей присутствовали рефлексы, относящиеся к $\mathrm{BaZrO}_{3-\delta}$.

Сложные оксиды $\mathrm{SmBaFe}{ }_{2-x} \mathrm{Cu}_{x} \mathrm{O}_{6-\delta}$

Твердые растворы состава $\mathrm{SmBaFe}_{2-x} \mathrm{CuxO}_{6-\delta}$, где $0,5 \leq x \leq 1,5$ с шагом 0,1 были синтезированы по глицерин-нитратной технологии. Согласно рентгенографическим данным твердые растворы $\mathrm{SmBaFe}_{2-x} \mathrm{Cu}_{x} \mathrm{O}_{6-\delta}$ с $0,7 \leq x \leq 1,3$ являются однофазными. Рентгенограммы оксидов $\mathrm{SmBaFe}_{2-x} \mathrm{Cu}_{x} \mathrm{O}_{6-\delta} \quad$ удовлетворительно описываются в рамках тетрагональной ячейки пространственной группы P4/ $m m m$. Пример рентгенограммы твердого раствора $\mathrm{SmBaFe}_{1.2} \mathrm{Cu}_{0.8} \mathrm{O}_{6-\delta}$, обработанной по методу полнопрофильного анализа Ритвелда, приведен на рис. 3.

Для всех однофазных оксидов были рассчитаны параметры элементарной ячейки и координаты атомов. Установлено, что при увеличении концентрации меди в $\mathrm{SmBaFe}_{2-x} \mathrm{Cu}_{x} \mathrm{O}_{6-\delta}$ приводит к постепенному увеличению параметров и объема элементарной ячейки, что можно объяснить с точки зрения размерных факторов. 


\section{Выводы}

По результатам проделанной работы можно сделать следующие выводы:

1. Определены области гомогенноститвердыхрастворов $\mathrm{SmBaFe}{ }_{2-x} \mathrm{Cu}_{x} \mathrm{O}_{6-\delta}$ $(0,7 \leq x \leq 1,3), \quad \mathrm{SmBaCo}_{2-x} \mathrm{Cu}_{x} \mathrm{O}_{6-\delta}$ $(0 \leq x \leq 1,2)$ на воздухе. Построены зависимости параметров элементарных ячеек от состава. Показано, что при увеличении степени замещения кобальта и железа на медь происходит монотонное увеличение параметров и объема элементарных ячеек в оксидах $\mathrm{SmBaMe}_{2-x} \mathrm{Cu}_{x} \mathrm{O}_{6-\delta}$.

2. Установлено, что твердые растворы $\mathrm{SmBaCo}_{2-x} \mathrm{Cu}_{x} \mathrm{O}_{6-\delta}$ в интервале составов $0 \leq x \leq 0,1$ кристаллизуются в орторомбической ячейке (пр. гр. Рmmm), а сложные оксиды $\mathrm{SmBaFe}_{2-x} \mathrm{CuxO}_{6-\delta}(0,7 \leq x \leq 1,3)$ и $\mathrm{SmBaCo}_{2-x} \mathrm{Cu}_{x} \mathrm{O}_{6-\delta}(0,2 \leq x \leq 1,2)-$ в тетрагональной (пр. гр. $P 4 / \mathrm{mmm}$ ).

3. Методами высокотемпературной термогравиметрии и йодометрического титрования определены значения содержания кислорода в сложных оксидах, образующихся в системах $\mathrm{Sm}-\mathrm{Ba}-\mathrm{Co}-\mathrm{Cu}-\mathrm{O}$ в широком интервале температур на воздухе. Установлено, что введение меди уменьшает содержание кислорода в $\mathrm{SmBaCo}_{2-x} \mathrm{Me}_{x} \mathrm{O}_{6-\delta}$.

4. Рассчитаны коэффициенты термического расширения (КТР) твердых

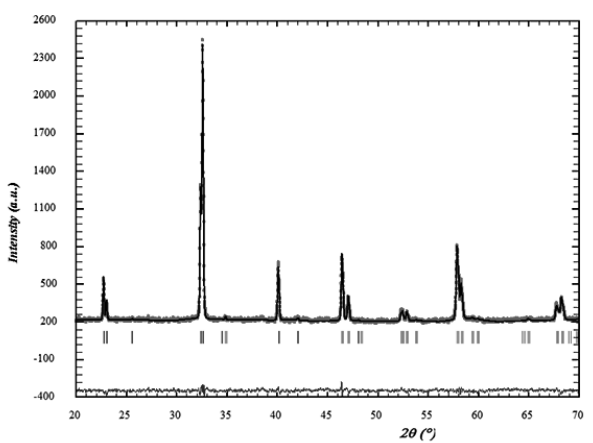

Рис. 3. Рентгеновские данные для $\mathrm{SmBaFe}_{1.2} \mathrm{Cu}_{0.8} \mathrm{O}_{6-\delta}$, обработанные по методу Ритвелда

растворов $\mathrm{SmBaCo}_{2-x} \mathrm{Cu}_{x} \mathrm{O}_{6-\delta} \quad(x=0$; $0,4 ; 0,6)$. Показано, что величина КТР уменьшается с увеличением концентрации меди в образцах.

5. Исследована химическая совместимость сложных оксидов с материалом твердого электролита $\left(\mathrm{Ce}_{0,8} \mathrm{Sm}_{0,2} \mathrm{O}_{2-\delta} \quad\right.$ и $\left.\mathrm{Zr}_{0,85} \mathrm{Y}_{0,15} \mathrm{O}_{2-\delta}\right)$ при $900 \leq \mathrm{T},{ }^{\circ} \mathrm{C} \leq 1100$ и $\mathrm{PO}_{2}=0,21$ атм. Показано, что твердые растворы состава $\mathrm{SmBaCo}_{2-x} \mathrm{Me}_{x} \mathrm{O}_{6-\delta}$ взаимодействуют со стабилизированным оксидом циркония уже при температуре $900{ }^{\circ} \mathrm{C}$ и не взаимодействуют со стабилизированным оксидом церия до температур не выше 900-1100 ${ }^{\circ} \mathrm{C}$ в зависимости от концентрации ионов меди.

1. Maignan A., Martin C., Pelloquin D., Nguyen N., Raveau B. Structural and Magnetic Studies of Ordered Oxygen - Deficient Perovskites $\mathrm{LnBaCo}_{2} \mathrm{O}_{5+\delta}$, Closely Related to the "112" Structure // J. Solid State Chem. 1999. V. 142. P. 247-260.

2. Anderson P. S., Kirk C. A., Knudsen J., Reaney I. M. West A. R. Structural characterization of $\mathrm{REBaCo}_{2} \mathrm{O}_{6-\delta}$ phases $(\mathrm{RE}=\mathrm{Pr}, \mathrm{Nd}, \mathrm{Sm}, \mathrm{Eu}, \mathrm{Gd}, \mathrm{Tb}, \mathrm{Dy}, \mathrm{Ho}) / / \mathrm{J}$. Solid State Scien. 2005. V. 7. P. 1149-1156.

3. Zhang K., Ge L., Ran R., Shao Z., Lio S. Synthesis, characterization and evaluation of cation-ordered $\mathrm{LnBaCo}_{2} \mathrm{O}_{5+\delta}$ as materials of oxygen permiation membranes and cathodes of SOFCs // Acta Mater. 2008. V. 56. P. 4876-4889. 
4. Shannon R. D. Revised effective ionic radii and systematic studies of interatomic distances in halides and chalcogenides // Acta Cryst. 1976. A. 32. P. 751-767.

5. Kini N. S., Shivashankar S. A., Umarji A. M., Yelon W. B., Malik S. K. Effect of synthesis route on the structure of $\mathrm{Nd}_{3-\mathrm{x}} \mathrm{Ba}_{3+\mathrm{x}} \mathrm{Cu}_{6} \mathrm{O}_{14-2 \delta}(\mathrm{x}=0,1)$ system: a neutron diffraction study // Solid State Comm. 2002. V. 122. P. 99-104.

6. Wende C., Schüpp B., Krabbes G. Phase equilibria and primary crystallisation field for $\mathrm{Sm}_{1+\mathrm{y}} \mathrm{Ba}_{2-\mathrm{y}} \mathrm{Cu}_{3} \mathrm{O}_{7}$ at various $\mathrm{p}(\mathrm{O} 2) / / \mathrm{J}$. Alloys and Comp. 2004. V. 381. P. 320 326.

Рекомендуем при цитировании данно статьи следующую ссылку: Volkova N. Е., Deryabina K. M., Gavrilova L. Ya., Cherepanov V. A. Physicochemical properties of the complex oxides in Sm-Ba-Me-Cu-O $(\mathrm{Me}=\mathrm{Fe}, \mathrm{Co})$ systems / Chimica Techno Acta. 2015. Vol. 2. № 2. P. 147-157. 